\title{
ANÁLISE DO COMPORTAMENTO COM RELAÇÃO AO ISOLAMENTO SOCIAL DA POPULAÇÃO DE ITAQUI - RS
}

\author{
ANALYSIS OF BEHAVIOR IN RELATION TO THE SOCIAL ISOLATION OF THE \\ POPULATION OF ITAQUI - RS
}

\author{
Dieison Morozoli da Silva ${ }^{1}$, Taináh Espinosa², Kellen da Rosa Carlosso ${ }^{3}$, Felipe \\ Batista Ethur ${ }^{4}$ e Luciana Zago Ethur ${ }^{5}$
}

1 Universidade Federal do Pampa, Brasil, E-mail: dieison.ufp@gmail.com, ORCID: https://orcid.org/0000-0001-5281-8427

2 Universidade Federal do Pampa, Brasil, E-mail: tainahespinosa.aluno@unipampa.edu.br, Lattes: http://lattes.cnpq.br/5126194225664962

3 Universidade Federal do Pampa, Brasil, E-mail: kellencarlosso.aluno@unipampa.edu.br, Lattes: http://lattes.cnpq.br/8456980561897183

4 Universidade Federal do Pampa, Brasil, E-mail: felipeethur@unipampa.edu.br, ORCID: https://orcid.org/0000-0003-4709-1379

5 Universidade Federal do Pampa, Brasil, E-mail: lucianaethur@unipampa.edu.br, ORCID: https://orcid.org/0000-0001-6664-8326

\section{ARTICLE INFO}

Article history:

Received 2020-09-23

Accepted 2020-12-12

Available online 2020-12-12
Palavras-chave: Covid-19. Mídias sociais. Saúde pública. Estresse.

Keywords: Covid-19. Social media. Public health. Stress.

RESUMO. A pandemia de coronavírus (Covid-19) motivou medidas de isolamento social (quarentena), que trouxeram mudanças consideráveis à rotina da população em geral. No que concerne ao ser humano enquanto integrante de uma sociedade, a realização de um isolamento social em massa requer uma ampla compreensão dos reflexos desse distanciamento. Assim, este trabalho objetivou investigar os impactos do isolamento social sobre o comportamento da população de Itaqui - RS, no período inicial do isolamento social recomendado pelo Ministério da Saúde. Itaqui, situada na fronteira oeste do Rio Grande do Sul, é a cidade mais distante da capital do Estado. A pesquisa constou de um questionário semiestruturado, com aplicação remota por redes sociais. Foram analisadas duas questões relacionadas à fonte e natureza das informações recebidas e duas relacionadas às vivências e aos hábitos no período do isolamento. Identificouse que se sobressaem como fonte de informações para a população itaquiense a televisão, que atinge $49,59 \%$ dos respondentes, e os sites da internet, com 31,78\%. Com relação ao estado emocional, manifestaram-se majoritariamente estressados e angustiados (61,65\%). Isso demonstra que, mesmo em uma cidade distante de grandes centros urbanos, a tensão sobre um tema tão preocupante atinge a maioria da população. E com relação ao que mudou na sua rotina, 40,27\% apontou que foi o isolamento social/familiar; $30,14 \%$ a higiene e $8,77 \%$ a rotina profissional ou estudantil. Pôde-se observar a desacomodação gerada pelo novo coronavírus, que interferiu em diversos aspectos da vida da maioria da população itaquiense, à semelhança da população brasileira e mundial.

ABSTRACT. The coronavirus pandemic (Covid-19) motivated measures of social isolation (quarantine), which brought considerable changes to the routine of the general population. With regard to the human being as a member of a society, the realization of mass social isolation requires a broad understanding of the consequences of this distance. Thus, this study aimed to investigate the impacts of social isolation on the behavior of the population of Itaqui - RS, in the initial period of social isolation recommended by the Ministry of Health. Itaqui, located on the western border of Rio Grande do Sul, is the most distant city of the state capital. The survey consisted of a semi-structured questionnaire, with 
remote application through social networks. Two questions related to the source and nature of the information received and two related to the experiences and habits during the isolation period were analyzed. It was identified that television, which reaches $49.59 \%$ of respondents, stands out as a source of information for the Itaquiense population, and internet sites, with $31.78 \%$. Regarding the emotional state, they were mostly stressed and distressed (61.65\%). This demonstrates that, even in a city far from large urban centers, tension on such a worrying issue affects the majority of the population. Regarding what changed in their routine, $40.27 \%$ pointed out that it was social / family isolation; $30.14 \%$ to hygiene and $8.77 \%$ to professional or student routine. It was possible to observe the discomfort caused by the new coronavirus, which interfered in several aspects of the life of the majority of the population of Itaquiense, similarly to the Brazilian and world population.

\section{Introdução}

A pandemia em escala mundial do novo coronavírus (Covid-19) causou e continua a causar impactos altamente sentidos nos mais diversos setores da sociedade. Em um curto espaço de tempo sua transmissão se sucedeu em larga escala, independentemente das fronteiras, classes sociais ou faixa etária.

Conforme Barreto et al. (2020) a pandemia por Covid-19 representa um dos maiores desafios sanitários em escala mundial desse século, de tal forma que na primeira semana do mês de abril, poucos meses depois do início da epidemia na China, em dezembro de 2019, já foram reportados mais de 1,5 milhão de casos e 85 mil mortes no mundo, e esperase que um número ainda maior de casos e óbitos venha a ocorrer nos próximos meses. Segundo os mesmos autores, no Brasil, até o dia oito de abril de 2020, foram registrados 15.927 casos confirmados e 800 mortes pelo Covid-19.

Em virtude da alta capacidade de propagação do Covid-19, uma das principais medidas adotadas com o intuito de mitigar os expressivos números de casos positivos foi o isolamento social. Para Spadacio \& Mello Alves (2020), o isolamento social é uma das principais medidas para frear a pandemia.

Segundo Oliveira et al. (2020), com o crescimento do número de casos da Covid-19 e a ocorrência de transmissão comunitária, estratégias de mitigação passaram a ser adotadas, buscando-se evitar a ocorrência de casos graves e óbitos pela doença, o que inclui medidas de atenção hospitalar para os casos graves, além de medidas de isolamento para casos leves e contatos.

Em vista da importância da prevenção à saúde e para o enfrentamento do Covid-19, foi publicada a Portaria no 356/2020 (BRASIL, 2020) pelo Ministério da Saúde, indicando a necessidade da adoção do isolamento social (quarentena).

Em um país de dimensões continentais como o Brasil, é notória a presença de diferentes culturas, condições econômicas, bem como desigualdades sociais e diferentes 
níveis de formação e informação dentre os cidadãos. No que concerne ao ser humano enquanto integrante de uma sociedade, a realização de um isolamento social em massa requer uma ampla compreensão dos reflexos desse distanciamento.

É importante que se tragam à lume os impactos mais expressivos do distanciamento, dado que em alguns casos as mudanças podem incorrer em episódios de estresse, incertezas ou mesmo degradação da condição humana devido a alterações bruscas, não planejadas e também muitas vezes indesejadas nas rotinas pessoais e de convivência familiar.

O município de Itaqui integra a fronteira oeste do Estado do Rio Grande do Sul, sendo separado pelo Rio Uruguai da cidade de Alvear, na Argentina. Possui como limites gaúchos os municípios de Alegrete, Maçambará, Manoel Viana, São Borja e Uruguaiana. Itaqui é a cidade mais distante da capital do Estado, Porto Alegre $(670 \mathrm{Km})$, dentre todas do Rio Grande do Sul. De acordo com IBGE (2020), a população estimada em 2019 era de 37.620 pessoas.

Com o intuito de saber como se comportava a população de Itaqui e quais fatores impactavam sua nova condição de vida durante o período inicial da pandemia, constituiu-se o Grupo de Pesquisa sobre Impacto Social do Coronavírus (Covid-19) do Campus Itaqui da UNIPAMPA, que realizou o presente trabalho de forma remota, com o objetivo de identificar e analisar as vivências, a mudança comportamental e as percepções da população da cidade de Itaqui - RS, em relação à Covid-19, no período inicial do isolamento social recomendado pelo Ministério da Saúde.

\section{Material e métodos}

A pesquisa foi realizada através da aplicação de um questionário semiestruturado com vinte e duas perguntas criado no ambiente Google Drive. As perguntas foram elaboradas com base no objetivo de analisar o comportamento da população de Itaqui (RS), em relação ao Covid-19, sendo que o formulário ficou disponibilizado no período de 02 até 11/04/2020. Cabe ressaltar que neste trabalho foram analisadas quatro questões: "A respeito do coronavírus (Covid-19), você costuma se informar principalmente através:"; "Você pensa que as notícias sobre o coronavírus (Covid-19) são:"; "O atual período que você está vivenciando está sendo:"; "O que mudou nos seus hábitos, na sua rotina, ou seja, no seu dia a dia, desde que surgiu o coronavírus?".

Para a realização da atividade, primeiramente foi criada a identidade visual do grupo de Pesquisa GPISC (Figura 1), que foi utilizada junto a todas as campanhas para estimular que os internautas respondessem o questionário. Além disso, foi criado um perfil no 
Instagram, para divulgação e contato com a comunidade. Após a organização do questionário e da logomarca do grupo ocorreu o lançamento da pesquisa aos internautas, utilizando-se o Facebook, Instagram e grupos de WhatsApp dos integrantes do Grupo de Pesquisa.

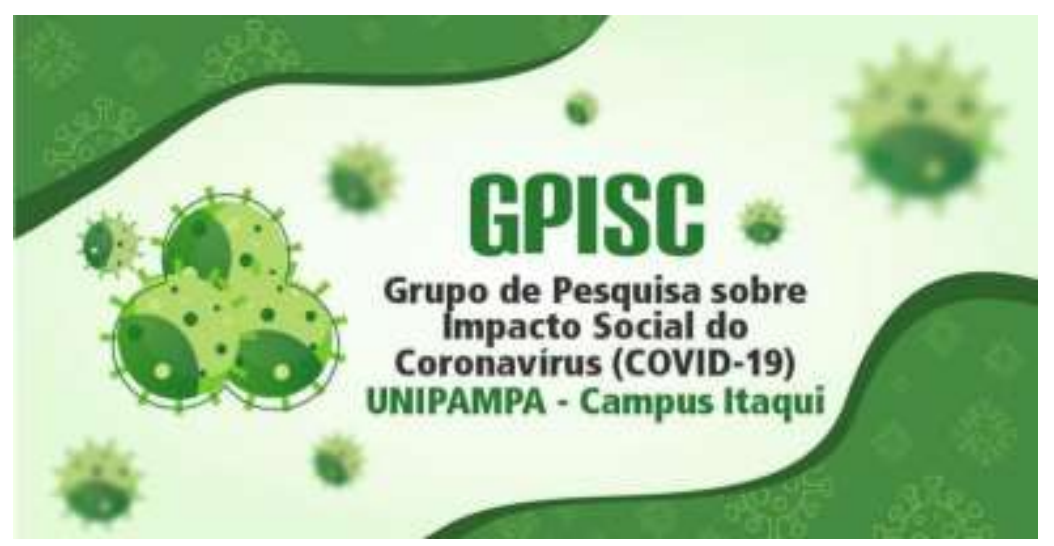

Figura 1 - Logomarca do grupo de pesquisa GPISC.

Fonte: os autores (2020)

Foram realizadas diferentes campanhas indicando e solicitando aos internautas que respondessem o questionário, tanto no Facebook quanto no Instagram que se prolongou até a última semana em que o link do formulário ficou disponível para ser respondido.

De posse dos dados coletados, as questões de interesse foram analisadas em duas etapas. A primeira etapa constituiu-se da elaboração de uma tabela com as respostas, quando pode-se identificar a percepção dos internautas com relação a pandemia causada pelo novo coronavírus. Na segunda etapa as respostas foram relacionadas a trabalhos e notícias publicadas sobre o tema, de acordo com a revisão de literatura realizada através do Google acadêmico e na base de dados da Scientific Electronic Library Online (Scielo), além da mídia digital. Isso foi realizado com o objetivo de compreender se a percepção da população de Itaqui e seu comportamento estão de acordo com o ocorrido de forma geral e se existem peculiaridades que devam ser salientadas.

\section{Resultados e discussão}

No total foram analisadas 365 respostas de itaquienses ao questionário, onde todas confirmaram o consentimento à realização da pesquisa e das quais extraíram-se as seguintes informações (Tabela 1). 
Tabela 1. Identificação do comportamento sobre o isolamento social devido a pandemia do novo coronavírus (Covid-19), dos 365 respondentes do questionário aplicado a internautas da cidade de Itaqui - RS, no período de 02 a 11/04/2020.

\begin{tabular}{|c|c|c|}
\hline Questão & Alternativas & Número - \% \\
\hline \multirow{5}{*}{$\begin{array}{l}\text { A respeito do coronavírus (Covid- } \\
\text { 19), você costuma se informar } \\
\text { principalmente através:" }\end{array}$} & Canais da televisão & $181-49,59$ \\
\hline & Sites da internet & $116-31,78$ \\
\hline & Redes sociais & $53-14,52$ \\
\hline & Emissoras de rádio & $13-3,56$ \\
\hline & Revistas e jornais impressos & $2-0,55$ \\
\hline \multirow{4}{*}{$\begin{array}{l}\text { Você pensa que as notícias sobre } \\
\text { o coronavírus (Covid-19) são:" }\end{array}$} & Reais, verdadeiras & $287-78,63$ \\
\hline & Alarmistas, exageradas & $60-16,44$ \\
\hline & Mal-intencionadas & $18-4,93$ \\
\hline & Falsa & $0-0$ \\
\hline \multirow{7}{*}{$\begin{array}{l}\text { O atual período que você está } \\
\text { vivenciando está sendo: }\end{array}$} & Estressante, angustiante & $225-61,65$ \\
\hline & Tranquilo & $59-16,16$ \\
\hline & Normal & $54-14,80$ \\
\hline & Agitado & $24-6,58$ \\
\hline & Atípico & $1-0,27$ \\
\hline & Necessário & $1-0,27$ \\
\hline & Outros & $1-0,27$ \\
\hline \multirow{6}{*}{$\begin{array}{l}\text { "O que mudou nos seus hábitos, } \\
\text { na sua rotina, ou seja, no seu dia a } \\
\text { dia, desde que surgiu o } \\
\text { coronavírus?" }\end{array}$} & Isolamento social/familiar & $147-40,27$ \\
\hline & Higiene & $110-30,14$ \\
\hline & Rotina profissional/estudante & $32-8,77$ \\
\hline & Estado emocional & $6-1,64$ \\
\hline & Sedentarismo & $6-1,64$ \\
\hline & Não houve mudanças & $64-17,54$ \\
\hline
\end{tabular}

Fonte: Os autores (2020). 
Notadamente, os meios de comunicação que se sobressaem como fonte de informações para a população itaquiense, acerca da Covid-19, são a televisão, que atinge $49,59 \%$ da população entrevistada, e os sites da internet, com $31,78 \%$. Observou-se que a televisão, mesmo em tempos de dispositivos com acesso a internet, persiste como um dos principais meios de comunicação, ocasionando inclusive forte popularização do assunto. Não para menos, conforme Barbosa Filho (2020), em tempos de isolamento social as maiores audiências têm apresentado pontuações recordes, ainda que considerados grandes eventos televisionados como Copa do Mundo de Futebol e Olimpíadas. Cabe ressaltar que o autor também destaca a televisão como um meio de comunicação presente em $92 \%$ dos lares brasileiros, além de ser considerado o meio de comunicação mais confiável por $79 \%$ da população. No mesmo sentido, e em termos retrospectivos, Salgueiro (2020) relata que Theodor Adorno (filósofo e sociólogo alemão), nos anos 1960, no artigo 'Televisão e formação', apontava: "existe uma espécie de função formativa ou deformativa operada pela televisão em relação à consciência das pessoas, conforme somos levados a supor a partir da enorme quantidade de espectadores e da enorme quantidade de tempo gasto vendo e ouvindo televisão“. Tais citações confirmam a enorme influência da televisão e seu papel sobre a consciência dos cidadãos através dos tempos.

Chama a atenção que os respondentes, mesmo sendo usuários de redes sociais, não apontaram como fonte principal de informações essas redes. Somente $14,52 \%$ afirmou se referenciar nelas. Isso é coerente com o resultado de uma pesquisa nacional do Instituto Datafolha (MARQUES, 2020), na qual consta que: "Em posição oposta à imprensa profissional estão os conteúdos que vêm de WhatsApp e Facebook. Nas duas plataformas, apenas $12 \%$ dizem confiar em informações sobre o coronavírus". Com relação ao predomínio dos sites da internet sobre as redes sociais, pode-se dever este fenômeno a algumas hipóteses: uma que há diversos sites fidedignos, de organismos oficiais, de órgãos de imprensa, etc. e outra que comumente são postados links nas redes sociais, os quais também direcionam seus usuários para sites externos.

Sabe-se que em Itaqui, cidade de aproximadamente 38.000 habitantes, grande parte da população tem por hábito ouvir emissoras locais de rádio. No entanto, possivelmente por tratar-se aqui de um tema de relevância mundial, com cenários de situações em diferentes países, a televisão tenha adquirido seu maior status, seguida de sites da internet e as emissoras de rádio tenham sido tão pouco mencionadas.

Com relação à avaliação que fazem da fidedignidade das informações (segunda pergunta), os respondentes de Itaqui manifestaram forte confiança na veracidade das notícias e informações que recebem sobre coronavírus dos meios de comunicação, na medida em que somente $21,37 \%$ considerou tais notícias alarmistas, exageradas ou mesmo 
mal-intencionadas. Embora com esse percentual crítico ao "teor" das informações, ninguém, entretanto, apontou-as como falsas.

Com relação ao estado emocional (terceira pergunta), os respondentes de Itaqui se manifestaram majoritariamente estressados e angustiados $(61,65 \%)$ e agitados $(6,58 \%)$ no período inicial da pandemia, quando o questionário foi aplicado. Tranquilos foi a opção de $16,16 \%$ e normais foi outra opção preferida de $14,80 \%$. Isso demonstra que, mesmo em uma cidade distante de grandes centros urbanos, a tensão sobre um tema tão preocupante atinge a maioria da população.

E com relação ao que mudou na sua rotina desde o surgimento do novo coronavírus, $40,27 \%$ dos respondentes itaquienses apontou que foi o isolamento social/familiar; $30,14 \%$ que foi a higiene e $8,77 \%$ que foi a rotina profissional ou estudantil. Houve $17,54 \%$ que apontaram não ter havido mudanças. Também por essas respostas pode-se observar a desacomodação gerada pelo novo coronavírus, que interferiu em diversos aspectos da vida da maioria da população itaquiense, à semelhança da população brasileira e mundial.

Fatores como esses levantados neste trabalho, incluindo o estado emocional atual da população itaquiense, interferem negativamente na rotina, prejudicando a produtividade de trabalho mesmo para quem eventualmente tenha passado a desenvolver suas atividades em seu próprio domicílio (através de home office). Interferem porque comprometem o bemestar geral, a qualidade de vida e porque deixam a todos(as) ansiosos(as) com relação à própria saúde, de familiares e amigos(as). Depender de sorte compromete a tranquilidade e não satisfaz a ninguém.

Assim, embora o isolamento social seja de extrema importância para o sucesso da contenção do Covid-19, a mudança na forma em que se dão as interações incorre em impactos sobre a vida da população, inclusive no que diz respeito à percepção de como é o período vivenciado.

Um aspecto preocupante que tem sido apontado nesta nova realidade brasileira sob isolamento social é a intensificação da violência em relações familiares, motivada por fatores como o maior período de convivência, mas especialmente por agravamento de estresse e nervosismo, muitas vezes associado à perda parcial ou total de renda. A mulher tem sido a vítima principal. Como narra Marques (2020), houve um aumento de cerca de $17 \%$ no número de ligações com denúncias de violência contra a mulher durante o mês de março. Além disso, conforme o mesmo autor, no Rio de Janeiro, dados do plantão do Ministério Público Estadual revelam um aumento de $50 \%$ nos casos de violência doméstica já no primeiro final de semana após os decretos estaduais que propuseram o distanciamento social, sendo a maior parte das denúncias envolvendo violência contra a mulher. 
Itaqui, por não estar dissociada da realidade nacional, deve atentar preventivamente para que o estresse e a angústia vividos, e reconhecidos por sua população, não redundem em deterioração das relações familiares e nem sociais.

\section{Conclusão}

Considerando-se que Itaqui é uma pequena cidade situada distante dos grandes centros urbanos do Rio Grande do Sul, verificou-se por este trabalho que a população local se alimenta de informações sobre a pandemia principalmente por canais de televisão e que atribui a essas informações fidedignidade com a realidade. O período inicial da pandemia, no qual foi aplicado o questionário, foi descrito pela ampla maioria dos respondentes itaquienses como estressante e angustiante. Com relação a quais mudanças foram principais em suas rotinas, $70 \%$ afirmou que ocorreram com relação ao isolamento social/familiar e hábitos de higiene.

Comparados através da bibliografia consultada, identificou-se similaridade entre os respondentes itaquienses e a população brasileira tanto com relação às vivências, quanto com relação às mudanças comportamentais e percepções sobre a pandemia em seu período inicial no Brasil.

\section{Agradecimentos}

O grupo idealizador deste trabalho agradece a todos que dedicaram seu tempo para responder à pesquisa, bem como àqueles que auxiliaram na divulgação do link de acesso ao questionário.

\section{Referências}

BARBOSA FILHO, André. Comunicação e Covid-19. In: CASTRO, Daniel; Dal SENO, Danillo; POCHMANN, Marcio. (Org.). Capitalismo e a Covid-19. São Paulo: 2020. Disponível em: http://abet-trabalho.org.br/wp-content/uploads/2020/05/LIVRO.CapitalismoxCovid19.pdf. BARRETO, Mauricio Lima; et al. O que é urgente e necessário para subsidiar as políticas de enfrentamento da pandemia de COVID-19 no Brasil? Revista Brasileira de Epidemiologia, vol. 23, p. 1-4, 2020. https://doi.org/10.1590/1980-549720200032. 
BRASIL. Governo Federal. Ministério da Saúde. Portaria 356/2020, de 11/03/2020. Disponível em: <http://www.in.gov.br/en/web/dou/-/portaria-n-356-de-11-de-marco-de-2020247538346>. Acesso em: 24 jun. 2020.

IBGE. Município de Itaqui - RS, Brasil. Disponível em: <https://cidades.ibge.gov.br/brasil/rs/itaqui/panorama>. Acesso em: 25 jun. 2020.

MARQUES, Emanuele Souza et al. A violência contra mulheres, crianças e adolescentes em tempos de pandemia pela COVID-19: panorama, motivações e formas de enfrentamento. Cadernos de Saúde Pública, vol. 36, no. 4, p. e00074420, 2020. https://doi.org/10.1590/0102-311X00074420

MARQUES, J. TVs e jornais lideram índice de confiança em informações sobre coronavírus, diz Datafolha. Jornal Folha de São Paulo, São Paulo, 23 de mar. 2020. Disponível em: <https://www1.folha.uol.com.br/poder/2020/03/tvs-e-jornais-lideram-indicede-confianca-em-informacoes-sobre-coronavirus-diz-datafolha.shtml>. Acesso em: 15 abr. 2020.

OLIVEIRA, Wanderson Kleber de; DUARTE, Elisete; FRANCA, Giovanny Vinicius Araujo de; GARCIA, Leila Posenato. How Brazil can hold back COVID-19. Epidemiologia e Servicos de Saúde : revista do Sistema Único de Saúde do Brasil, vol. 29, n. 2, 2020. https://doi.org/10.5123/s1679-49742020000200023.

SALGUEIRO, W. Nova canção do exílio, de Luis Fernando Verissimo. Resenha. Revista Rascunho: o jornal de literatura do Brasil, n. 238, p. 18, fev. 2020.

SOUZA, D.O. A pandemia de COVID-19 para além das Ciências da Saúde: Reflexões sobre sua determinação social. Cien Saude Colet [periódico na internet] (2020/Abr). [Citado em 21/05/2020]. Disponível em: http://www.cienciaesaudecoletiva.com.br/artigos/apandemia-de-covid19-para-alem-das-ciencias-da-saude-reflexoes-sobre-sua-determinacaosocial/17562.

SPADACIO, Cristiane; ALVES, Márcia Guimarães de Mello. Nos entremeios: o biológico e o social no Brasil no contexto da COVID-19 e o papel da Atenção Primária à Saúde. Revista da Rede de Pesquisa em Atenção Primária à Saúde, vol. 2, n. 1, p. 61-65, 2020. https://doi.org/10.14295/aps.v2i3.67 